\title{
Cytotoxicity screening of cotreatment with temozolomide and azithromycin on human glioblastoma cells U87
}

\author{
Siti Nazihahasma Hassan ${ }^{1,4}$, Abdul Aziz Mohamed Yusoff ${ }^{1,2,4}$, Norhanani Mohd Redzwan ${ }^{3,4}$, Zamzuri Idris $^{1,4}$, \\ Farizan Ahmad ${ }^{1,2,4^{*}}$ \\ ${ }^{1}$ Department of Neurosciences, School of Medical Sciences, Universiti Sains Malaysia, 16150 Kubang Kerian Kelantan, Malaysia. \\ ${ }^{2}$ Human Genome Center, School of Medical Sciences, Universiti Sains Malaysia, 16150 Kubang Kerian, Kelantan, Malaysia. \\ ${ }^{3}$ Department of Immunology, School of Medical Sciences, Universiti Sains Malaysia, 16150 Kubang Kerian Kelantan, Malaysia. \\ ${ }^{4}$ Hospital Universiti Sains Malaysia, 16150 Kubang Kerian Kelantan, Malaysia.
}

\begin{tabular}{l}
\hline ARTICLE INFO \\
\hline Received on: $16 / 12 / 2020$ \\
Accepted on: 01/03/2021 \\
Available online: 05/04/2021 \\
\\
\hline Key words: \\
Temozolomide, azithromycin, \\
cotreatment, cytotoxicity, \\
glioblastoma cells.
\end{tabular}

\section{INTRODUCTION}

Temozolomide (TMZ) chemotherapy remains the keystone treatment modality for patients with glioblastoma (GBM). Although the administration of concomitant and adjuvant TMZ along with radiotherapy has achieved longer-term survival, its efficacy is limited by a wide range of aspects (Chen et al., 2012; Stupp et al., 2005). As such, numerous efforts are being made to improve the efficacy of TMZ and one of the current horizons is the combination of drugs. This approach to drug design has been supported by encouraging clinical benefits, such as sequential bortezomib and TMZ (Rahman et al., 2020).

\footnotetext{
"Corresponding Author

Farizan Ahmad, Department of Neurosciences, School of Medical Sciences, Universiti Sains Malaysia, Kubang Kerian, Malaysia; Human Genome Center, School of Medical Sciences, Universiti Sains Malaysia, 16150 Kubang Kerian, Kelantan, Malaysia; Hospital Universiti Sains Malaysia, 16150 Kubang Kerian Kelantan, Malaysia.E-mail: farizan@ usm.my
}

The nonantibiotic properties of azithromycin (AZI) have been shown to be active and effective against cancer cells and cancer stem-like cells (CSCs) of multiple human cancer types (Lamb et al., 2015; Qiao et al., 2018; Zhou et al., 2012). The combination of AZI and tumor necrosis factor-related apoptosisinducing ligand obtained synergistic cytotoxicity in colon cancer cells in vitro and in vivo (Qiao et al., 2018). AZI also has selective cytotoxicity to cancer cells and synergistic cytotoxicity with the chemotherapeutic agent vincristine in cervical and gastric cancer cells in vitro (Zhou et al., 2012). Besides, the 1-year survival rate of advanced non-small-cell lung cancer patients demonstrated an increase with the addition of AZI to the chemotherapy regimen (Chu et al., 2014).

Screening to understand the properties of drugs in combination is a fundamental element in the development of anticancer combination therapies. In this study, the cytotoxicity of cotreatment with TMZ and AZI on human GBM cells U87 was examined using the 3-(4,5-dimethylthiazol-2-yl)-2,5diphenyltetrazolium bromide (MTT) assay and drug interactions 
were analyzed by the Code for the Identification of Synergism Numerically Efficient (CISNE) software.

\section{MATERIALS AND METHODS}

\section{Drugs}

TMZ (CAS Number: 85622-93-1, Sigma-Aldrich, St. Louis, MO) and AZI (CAS Number: 83905-01-5, St. Louis, MO) were dissolved in dimethyl sulfoxide (CAS Number: 67-68-5, St. Louis, MO).

\section{Cell line}

The human GBM cells U87 MG (HTB-14 Lot number: 63710285) were purchased from the American Type Culture Collection (Rockville, MD). Cells were grown in Dulbecco's modified Eagle's medium containing glucose (4.5 g/l), sodium pyruvate, and L-glutamine (Nacalai Tesque, Japan), supplemented with $1 \%$ nonessential amino acids (Nacalai Tesque, Japan) and 5\% fetal bovine serum (Tico Europe, Netherlands) under standardized conditions $\left(5 \% \mathrm{CO}_{2}\right.$ at $37^{\circ} \mathrm{C} / 95 \%$ humidity).

\section{3-(4,5-dimethylthiazol-2-yl)-2,5-diphenyltetrazolium bromide (MTT) assay}

Five thousand cells with a final volume of $100 \mu$ were seeded in 96-well flat bottomed plates (SPL Life Sciences, Korea) and were incubated for 24 hours. Then, the cells were treated with TMZ, AZI, and a combination thereof in a series of concentrations $(0.5-225.0 \mu \mathrm{g} / \mathrm{ml})$. After 48 hours, MTT (Invitrogen, Thermo Fisher Scientific, Waltham, MA) assay was carried out as instructed by the manufacturer and the absorbance was taken at a wavelength of $540 \mathrm{~nm}$ using the Varioskan LUX multimode microplate reader (Thermo Fisher Scientific, Waltham, MA). The percentage of cell viability or a fraction of unaffected $(\mathrm{Fu})$ cells was calculated using the following formula:

(absorbance of treated Cell viability $(\%)=\frac{\text { cells }- \text { absorbance of blank })}{\begin{array}{c}\text { (absorbance of untreated } \\ \text { cells }- \text { absorbance of blank })\end{array}} \times 100$

\section{Drug combination analysis}

To determine the cytotoxic interactions between TMZ and AZI, CISNE software version 1.0 (Universidad de Oviedo, Spain) (García-Fuente et al., 2018) was used to compute the combination index (CI) and the isobologram. Synergistic, additive, and antagonistic interactions are indicated by CI values of $<0.8$, $0.8-1.2$, and $>1.2$, respectively. The isobologram represents the concentration of TMZ and AZI on the $x$ - and $y$-axis, respectively. A straight red line joins the half-maximal inhibitory concentration $\left(\mathrm{IC}_{50}\right)$ of each drug and dashed red lines demonstrate the upper and lower limits of a $95 \%$ confidence interval. The $\mathrm{IC}_{50}$ in the combination is plotted as a blue dot, with the confidence interval of both drug concentrations represented by a dashed blue line in an elliptical shape.

\section{RESULTS AND DISCUSSION}

Drug interactions are an important aspect of anticancer drug design. Scientists have gone the extra mile to achieve synergistic efficacy of various drug combinations because synergistic-acting combinations not only increase the effect but also decrease the dose. Besides, interactions between drugs in combination can also be classified as either antagonistic or additive. Antagonistic combinations generate lower effects than the additive because one drug hinders the efficacy of another. Alternatively, the additive occurs when the drugs do not interact with each other, and therefore, the effect is neither more nor less than individually (Chou, 2006; Zimmermann et al., 2007).

In the present study, cotreatment of the human GBM cells U87 with TMZ and AZI ameliorates the cytotoxicity in a dose-dependent manner (Fig. 1). However, the inhibitory effect improved slightly compared to TMZ alone, while it was markedly lower than AZI alone. The concentration of TMZ + AZI that caused $50 \%$ of the cells to be affected $\left(\mathrm{IC}_{50}\right)$ was $121.77 \pm 25.19 \mu \mathrm{g} / \mathrm{ml}$, compared to TMZ and AZI individually at $201.22 \pm 48.78 \mu \mathrm{g} /$ $\mathrm{ml}$ and $61.80 \pm 27.88 \mu \mathrm{g} / \mathrm{ml}$, respectively (Table 1 ). Specifically, drug interaction analysis evidenced an antagonistic interaction between TMZ and AZI (Fig. 2), which accounted for the observed competitive inhibition of cell viability (Fig. 1). This discovery, therefore, postulates that the alkylating agent (TMZ) and the
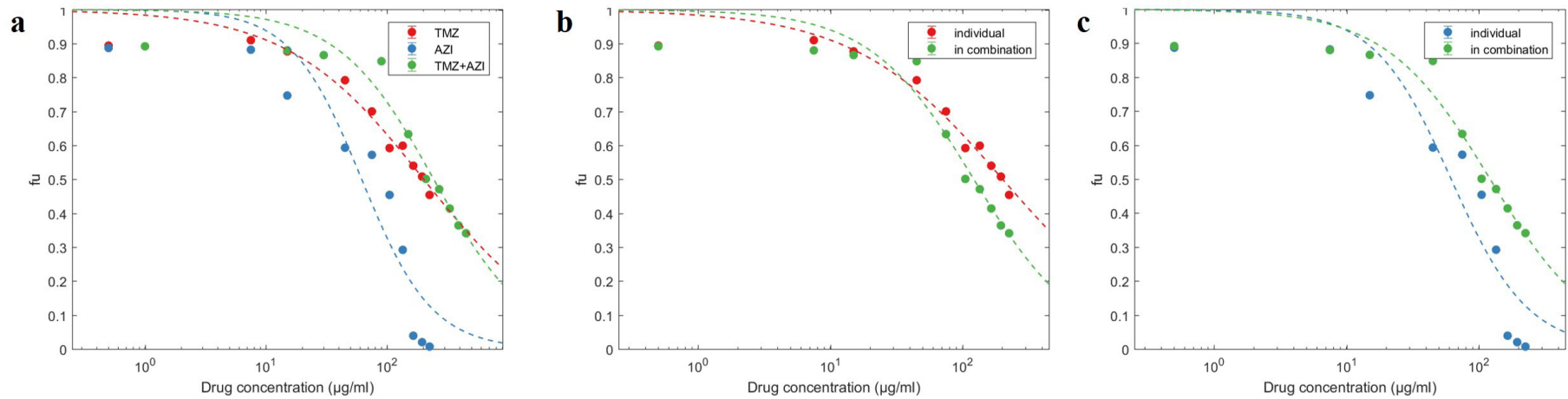

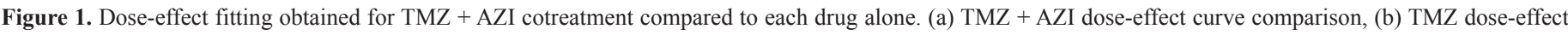

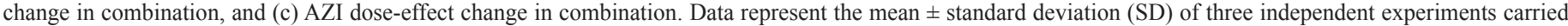
out in triplicate. 
Table 1. Antagonistic cytotoxicity of cotreatment with TMZ and AZI on U87 cells for 48 hours.

\begin{tabular}{|c|c|c|c|c|c|c|}
\hline \multirow{2}{*}{ TMZ $(\mu \mathrm{g} / \mathrm{ml})$} & \multirow{2}{*}{$\mathrm{AZI}(\mu \mathrm{g} / \mathrm{ml})$} & \multirow{2}{*}{$\mathbf{F u}$} & \multicolumn{2}{|c|}{ CISNE-generated parameters } & \multicolumn{2}{|c|}{ CISNE-simulation } \\
\hline & & & m & D & $\mathbf{F a}$ & CI \\
\hline 0.50 & & 0.89 & $0.77 \pm 0.22$ & $201.22 \pm 48.78$ & 0.05 & 2.87 \\
\hline 7.50 & & 0.91 & & & 0.10 & 2.59 \\
\hline 15.00 & & 0.88 & & & 0.15 & 2.49 \\
\hline 45.00 & & 0.79 & & & 0.20 & 2.45 \\
\hline 75.00 & & 0.70 & & & 0.25 & 2.45 \\
\hline 105.00 & & 0.59 & & & 0.30 & 2.45 \\
\hline 135.00 & & 0.60 & & & 0.35 & 2.47 \\
\hline 165.00 & & 0.54 & & & 0.40 & 2.50 \\
\hline 195.00 & & 0.51 & & & 0.45 & 2.53 \\
\hline \multirow[t]{11}{*}{225.00} & & 0.45 & & & 0.50 & 2.58 \\
\hline & 0.50 & 0.89 & $1.49 \pm 0.94$ & $61.80 \pm 27.88$ & 0.55 & 2.63 \\
\hline & 7.50 & 0.88 & & & 0.60 & 2.69 \\
\hline & 15.00 & 0.75 & & & 0.65 & 2.76 \\
\hline & 45.00 & 0.59 & & & 0.70 & 2.84 \\
\hline & 75.00 & 0.57 & & & 0.75 & 2.95 \\
\hline & 105.00 & 0.45 & & & 0.80 & 3.09 \\
\hline & 135.00 & 0.29 & & & 0.85 & 3.28 \\
\hline & 165.00 & 0.04 & & & 0.90 & 3.57 \\
\hline & 195.00 & 0.02 & & & 0.95 & 4.15 \\
\hline & 225.00 & 0.01 & & & & \\
\hline 0.25 & 0.25 & 0.89 & $1.10 \pm 0.39$ & $121.77 \pm 25.19$ & & \\
\hline 3.75 & 3.75 & 0.88 & & & & \\
\hline 7.50 & 7.50 & 0.87 & & & & \\
\hline 22.50 & 22.50 & 0.85 & & & & \\
\hline 37.50 & 37.50 & 0.63 & & & & \\
\hline 52.50 & 52.50 & 0.50 & & & & \\
\hline 67.50 & 67.50 & 0.47 & & & & \\
\hline 82.50 & 82.50 & 0.41 & & & & \\
\hline 97.50 & 97.50 & 0.36 & & & & \\
\hline 112.50 & 112.50 & 0.34 & & & & \\
\hline
\end{tabular}

a

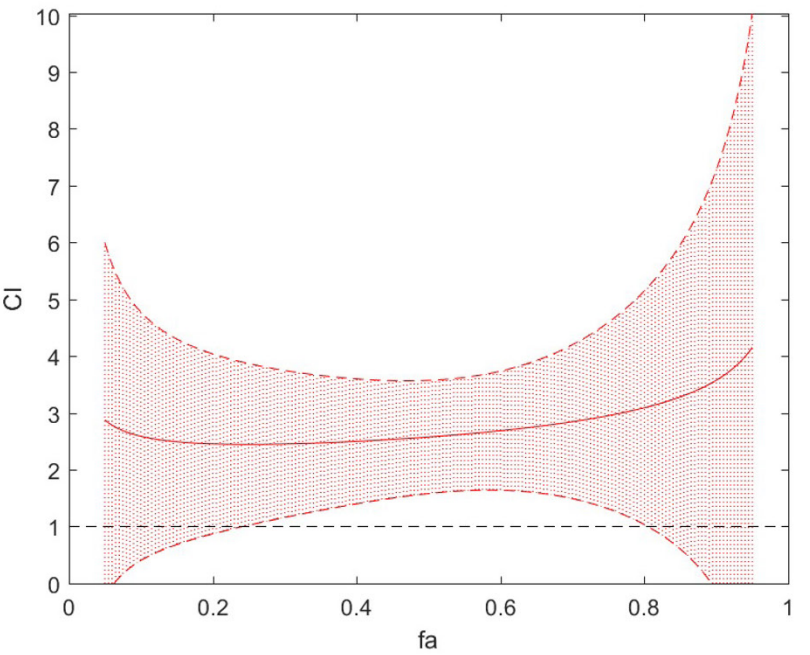

b

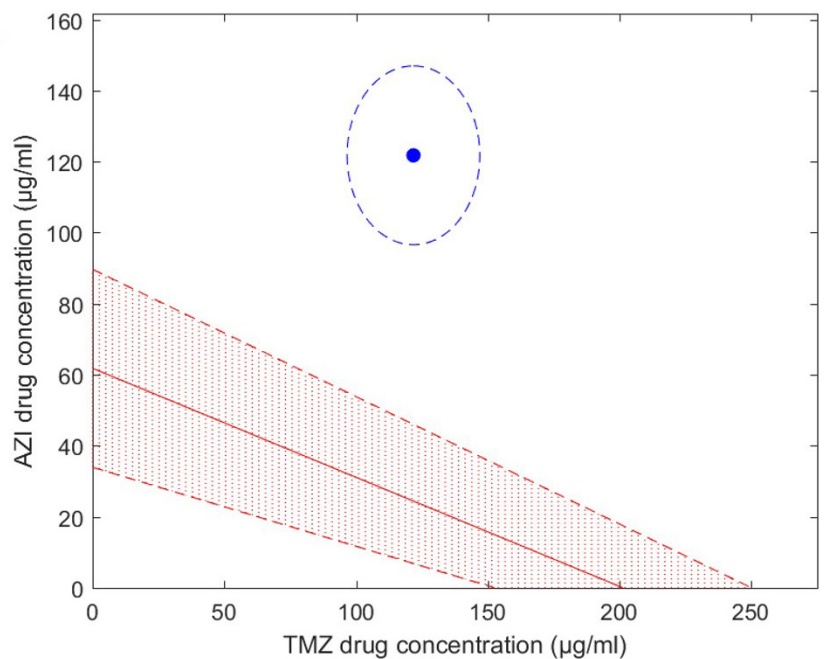

Figure 2. CISNE-generated (a) Fa-CI plot and (b) isobologram for TMZ + AZI cotreatment. The red line with the 95\% confidence interval (red-shaded region) in the Fa-CI plot above the black dashed line indicates an antagonistic interaction between TMZ and AZI. The isobologram, wherein the red line represents an additive effect, is synergistic if the blue dot within the space is limited by the red line and the axes, antagonistic if the blue dot is outside the triangle, and additive if the confidence intervals overlap. 
macrolide antibiotic (AZI) may compete for the same molecular (protein) target and cause cell death by the same mechanism in U87 cells. Indeed, studies have shown that TMZ and AZI can exert their cytotoxic activity, partly by mitochondrial-mediated caspase-dependent apoptosis (Lamb et al., 2015; Liu et al., 2018; Zhou et al., 2012). In a different study, the combination of TMZ and curcumin (a bioactive compound from turmeric) showed an additive effect on GBM cells in vitro and tumor growth in vivo due to redundant cytotoxicity mechanisms (Zanotto-Filho et al., 2015).

According to a competitive binding model, two drugs will be antagonistic if they target similar sites for the same protein (Krogstad et al., 1986). Drug combinations targeting the same protein have also been delineated as antagonistic companions (Cokol et al., 2011). Furthermore, a genetic epistatic interaction study suggests that two drugs will be synergistic if they inhibit different molecules or pathways of interconnected networks that serve the same purpose (Yeh et al., 2009). Certainly, synergisticacting combinations rarely have similar or directly linked targets characterized by a network-based computational method (Zou et al., 2012).

It is known that the use of drugs in combination can effectively control cancers by simultaneously thwarting a number of targets and making them less susceptible to resistance (Qiao et al., 2018; Yeh et al., 2009; Zimmermann et al., 2007). While synergistic efficacy is pressingly desired in this realm, drug combinations with antagonistic interaction may not be disregarded. In one study, the antagonistic combination is capable of offering a long-term constraint on the rise of therapeutic resistance compared to the synergistic combination (Saputra et al., 2018). Similarly, in the perspective of antimicrobials, antagonistic combinations can select antibiotic-resistant bacteria and minimize the evolution of resistance (Yeh et al., 2009). Herein, TMZ partially suppressed the cytotoxicity of AZI in U87 cells (Table 1) (see also Supplementary File 1). Taking into account the fact that AZI was found to be active and effective against both non-CSCs and CSCs, while TMZ was not (Chen et al., 2012; Lamb et al., 2015; Qiao et al., 2018; Zhou et al., 2012), it may be helpful to target therapy-resistant GBM by combining both drugs.

Additionally, sensitization is another strategy that can enhance the efficacy of anticancer therapy and reduce the side effects (Ciechomska et al., 2018; Matsuno et al., 2018). An in vitro investigation uncovered that pretreatment and posttreatment with a small-molecule inhibitor for the histone methyltransferase G9a (a biomarker associated with cancer progression and metastasis) sensitized GBM cells to TMZ and increased the efficacy of TMZ (Ciechomska et al., 2018). From now on, alternative ways, such as pretreatment, to evaluate whether AZI can sensitize cells to TMZ could be an approach worth exploring.

\section{CONCLUSION}

Cotreatment with TMZ and AZI exhibits antagonistic cytotoxicity in human GBM cells U87 between low and middle concentration ranges. This finding warrants further investigation using a number of different GBM cell lines, including glioma stem-like cells.

\section{FUNDING}

The authors would like to thank FRGS (203/ PPSP/6171203) provided by the Ministry of Higher Education
Malaysia, Postgraduate Funding Grant (311/PPSP/4404811), and Universiti Sains Malaysia Fellowship provided by the Universiti Sains Malaysia.

\section{AUTHOR CONTRIBUTIONS}

All authors made substantial contributions to conception and design, acquisition of data, or analysis and interpretation of data; took part in drafting the article or revising it critically for important intellectual content; agreed to submit to the current journal; gave final approval of the version to be published; and agree to be accountable for all aspects of the work. All the authors are eligible to be an author as per the international committee of medical journal editors (ICMJE) requirements/guidelines.

\section{CONFLICTS OF INTEREST}

The authors report no financial or any other conflicts of interest in this work.

\section{ETHICAL APPROVALS}

Not applicable.

\section{PUBLISHER'S NOTE}

This journal remains neutral with regard to jurisdictional claims in published institutional affiliation.

\section{REFERENCES}

Chen J, Li Y, Yu TS, McKay RM, Burns DK, Kernie SG, Parada LF. A restricted cell population propagates glioblastoma growth after chemotherapy. Nature, 2012; 488(7412):522.

Chou TC. Theoretical basis, experimental design, and computerized simulation of synergism and antagonism in drug combination studies. Pharmacol Rev, 2006; 58(3):621-81.

Chu D, Yao D, Zhuang Y, Hong Y, Zhu X, Fang Z, Yu J, Yu Z. Azithromycin enhances the favorable results of paclitaxel and cisplatin in patients with advanced non-small cell lung cancer. Genet Mol Res, 2014; 13(2):2796-805.

Ciechomska IA, Marciniak MP, Jackl J, Kaminska B. Pretreatment or post-treatment of human glioma cells with BIX01294, the inhibitor of histone methyltransferase G9a, sensitizes cells to temozolomide. Front Pharmacol, 2018; 9:1271.

Cokol M, Chua HN, Tasan M, Mutlu B, Weinstein ZB, Suzuki Y, Nergiz ME, Costanzo M, Baryshnikova A, Giaever G. Systematic exploration of synergistic drug pairs. Mol Syst Biol, 2011; 7(1):544.

García-Fuente A, Vázquez F, Viéitez JM, Alonso FJG, Martín JI, Ferrer J. CISNE: an accurate description of dose-effect and synergism in combination therapies. Sci Rep, 2018; 8(1): 1-9.

Krogstad D, Moellering R, Lorian, V. Antimicrobial combinations. In: Lorian V (ed.). Antibiotics in laboratory medicine. 2nd edition, The Williams \& Wilkins Co., Baltimore, MD, pp 537-595, 1986.

Lamb R, Ozsvari B, Lisanti CL, Tanowitz HB, Howell A, Martinez-Outschoorn UE, Sotgia F, Lisanti MP. Antibiotics that target mitochondria effectively eradicate cancer stem cells, across multiple tumor types: treating cancer like an infectious disease. Oncotarget, 2015; 6(7):4569.

Liu WJ, Yin YB, Sun JY, Feng S, Ma JK, Fu XY, Hou YJ, Yang MF, Sun BL, Fan CD. Natural borneol is a novel chemosensitizer that enhances temozolomide-induced anticancer efficiency against human glioma by triggering mitochondrial dysfunction and reactive oxide species-mediated oxidative damage. Onco Targets Ther, 2018; 11:5429.

Matsuno Y, Hyodo M, Fujimori H, Shimizu A, Yoshioka, KI. Sensitization of cancer cells to radiation and topoisomerase I inhibitor camptothecin using inhibitors of PARP and other signaling molecules. Cancers, 2018; 10(10):364. 
Qiao X, Wang X, Shang Y, Li Y, Chen SZ. Azithromycin enhances anticancer activity of TRAIL by inhibiting autophagy and upregulating the protein levels of DR4/5 in colon cancer cells in vitro and in vivo. Cancer Commun, 2018; 38(1):43.

Rahman MA, Brekke J, Arnesen V, Hannisdal MH, Navarro AG, Waha A, Herfindal L, Rygh CB, Bratland E, Brandal P. Sequential bortezomib and temozolomide treatment promotes immunological responses in glioblastoma patients with positive clinical outcomes: a phase 1B study. Immun Inflamm Dis, 2020; 8(3):342-59.

Saputra EC, Huang L, Chen Y, Tucker-Kellogg L. Combination therapy and the evolution of resistance: the theoretical merits of synergism and antagonism in cancer. Cancer Res, 2018; 78(9):2419-31.

Stupp R, Mason WP, Van Den Bent MJ, Weller M, Fisher B, Taphoorn MJ, Belanger K, Brandes AA, Marosi C, Bogdahn U. Radiotherapy plus concomitant and adjuvant temozolomide for glioblastoma. N Engl J Med, 2005; 352(10):987-996.

Yeh PJ, Hegreness MJ, Aiden AP, Kishony R. Drug interactions and the evolution of antibiotic resistance. Nat Rev Microbiol, 2009; $7(6): 460-466$.

Zanotto-Filho A, Braganhol E, Klafke K, Figueiró F, Terra SR, Paludo FJ, Morrone M, Bristot IJ, Battastini AM, Forcelini CM. Autophagy inhibition improves the efficacy of curcumin/temozolomide combination therapy in glioblastomas. Cancer Lett, 2015; 358(2):220-231.

Zhou X, Zhang Y, Li Y, Hao X, Liu X, Wang Y. Azithromycin synergistically enhances anti-proliferative activity of vincristine in cervical and gastric cancer cells. Cancers, 2012; 4(4):1318-32.

Zimmermann GR, Lehar J, Keith CT. Multi-target therapeutics: when the whole is greater than the sum of the parts. Drug Discov Today, 2007; 12(1-2):34-42.

Zou J, Ji P, Zhao YL, Li LL, Wei YQ, Chen YZ, Yang SY. Neighbor communities in drug combination networks characterize synergistic effect. Mol Biosyst, 2012; 8(12):3185-96.

\section{How to cite this article:}

Hassan SN, Yusoff AAM, Redzwan NM, Idris Z, Ahmad F. Cytotoxicity screening of cotreatment with temozolomide and azithromycin on human glioblastoma cells U87. J Appl Pharm Sci, 2021; 11(04):145-152. 


\section{Supplementary File 1.}
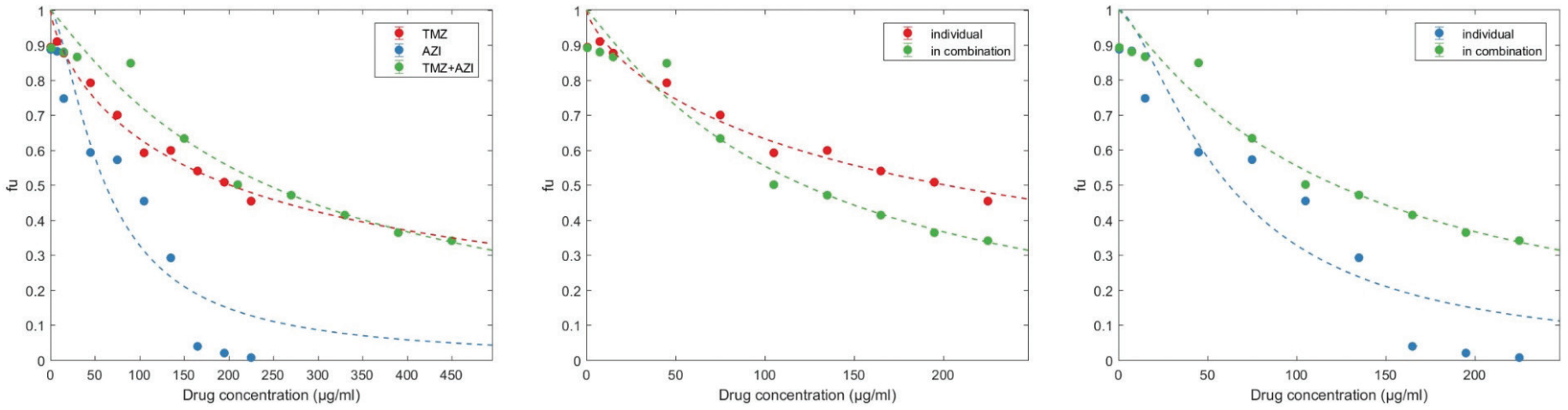

Figure 1. CISNE-generated dose-effect curve for TMZ + AZI cotreatment compared to each drug individually.
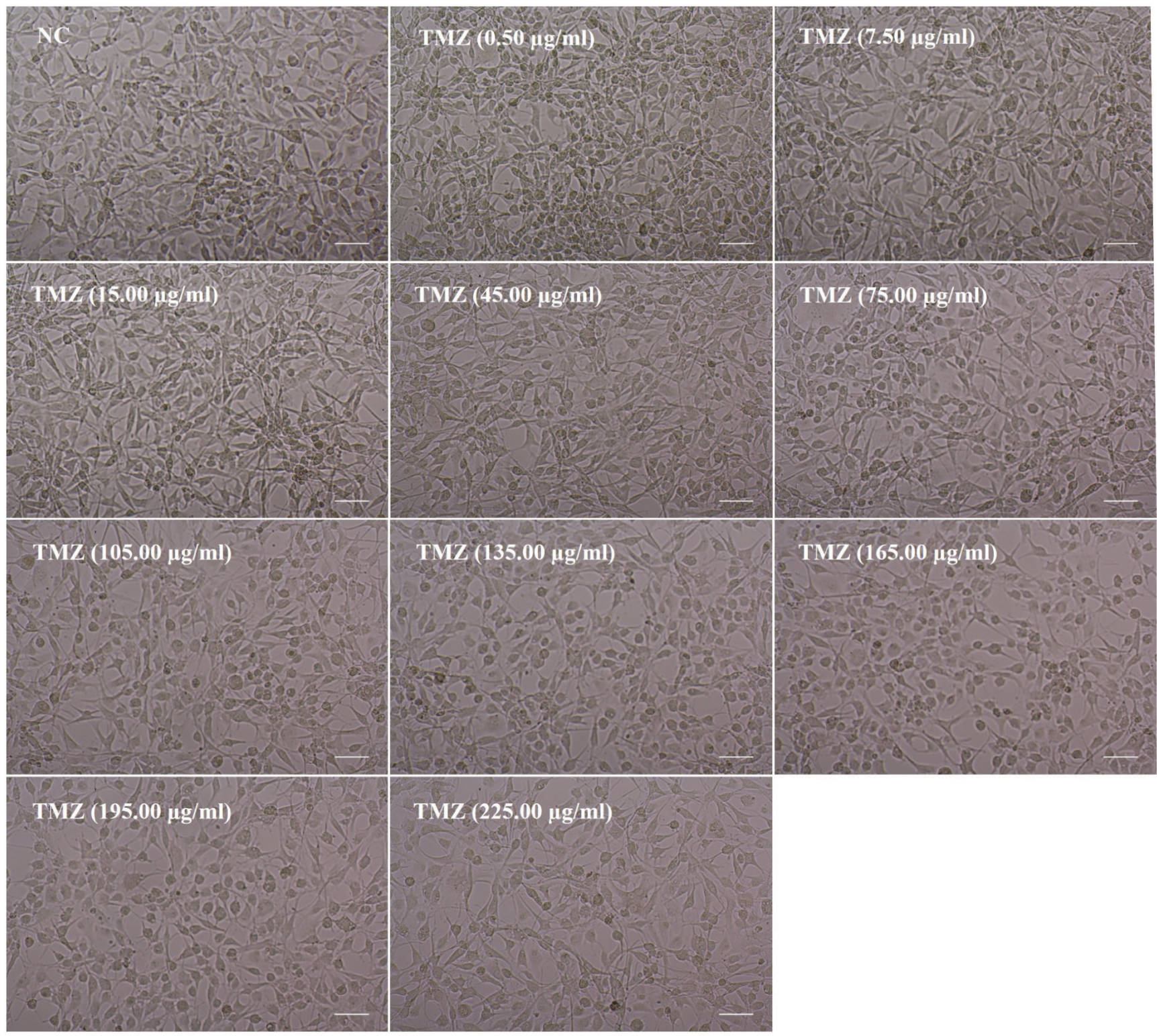

Figure 2. Effect of TMZ on U87 cells. Microphotographs were taken after 48h of incubation with different concentrations of TMZ (scale bars $=100 \mu \mathrm{m})$. 


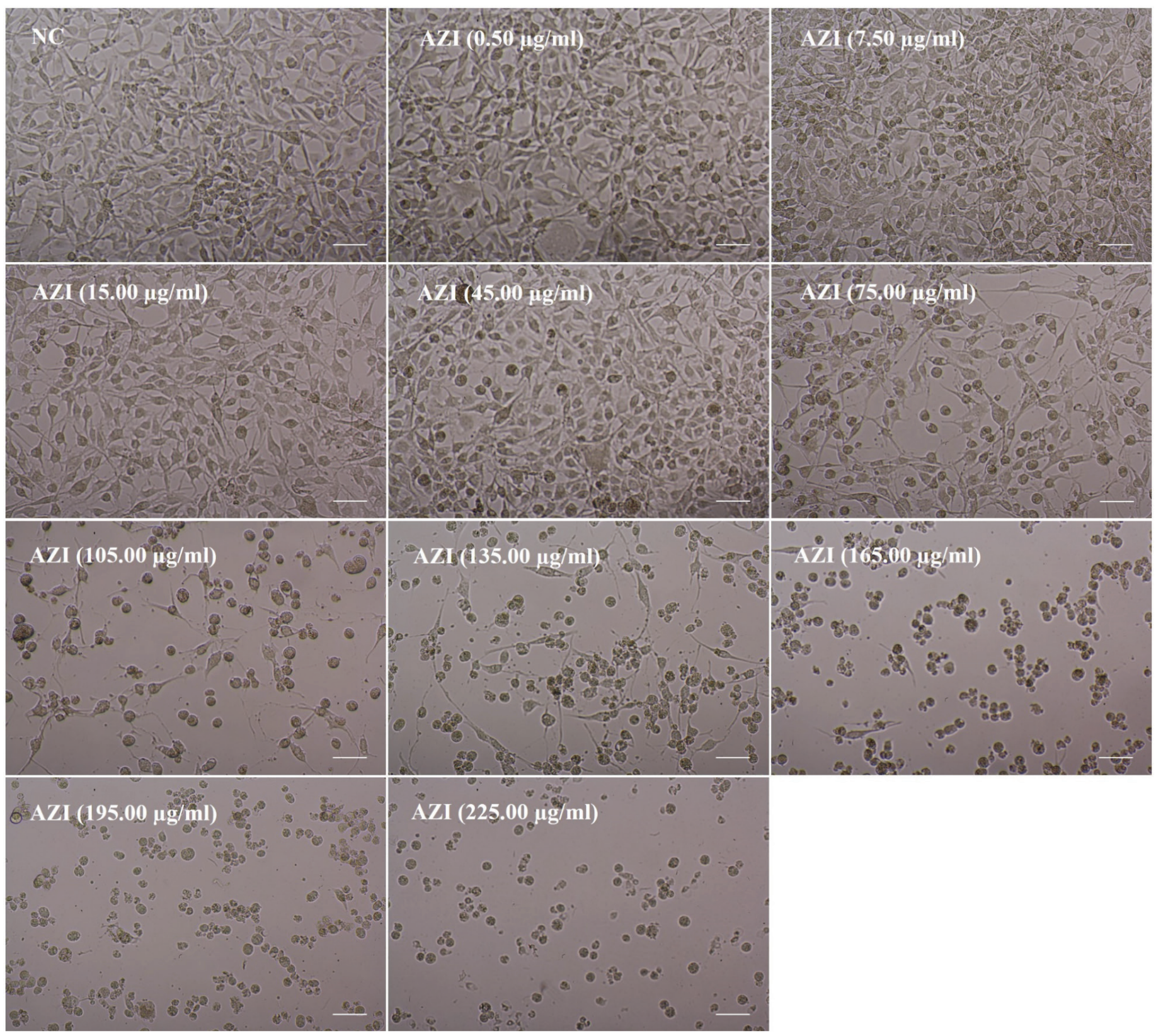

Figure 3. Effect of AZI on U87 cells. Microphotographs were taken after $48 \mathrm{~h}$ of incubation with different concentrations of AZI (scale bars $=100 \mu \mathrm{m})$. 

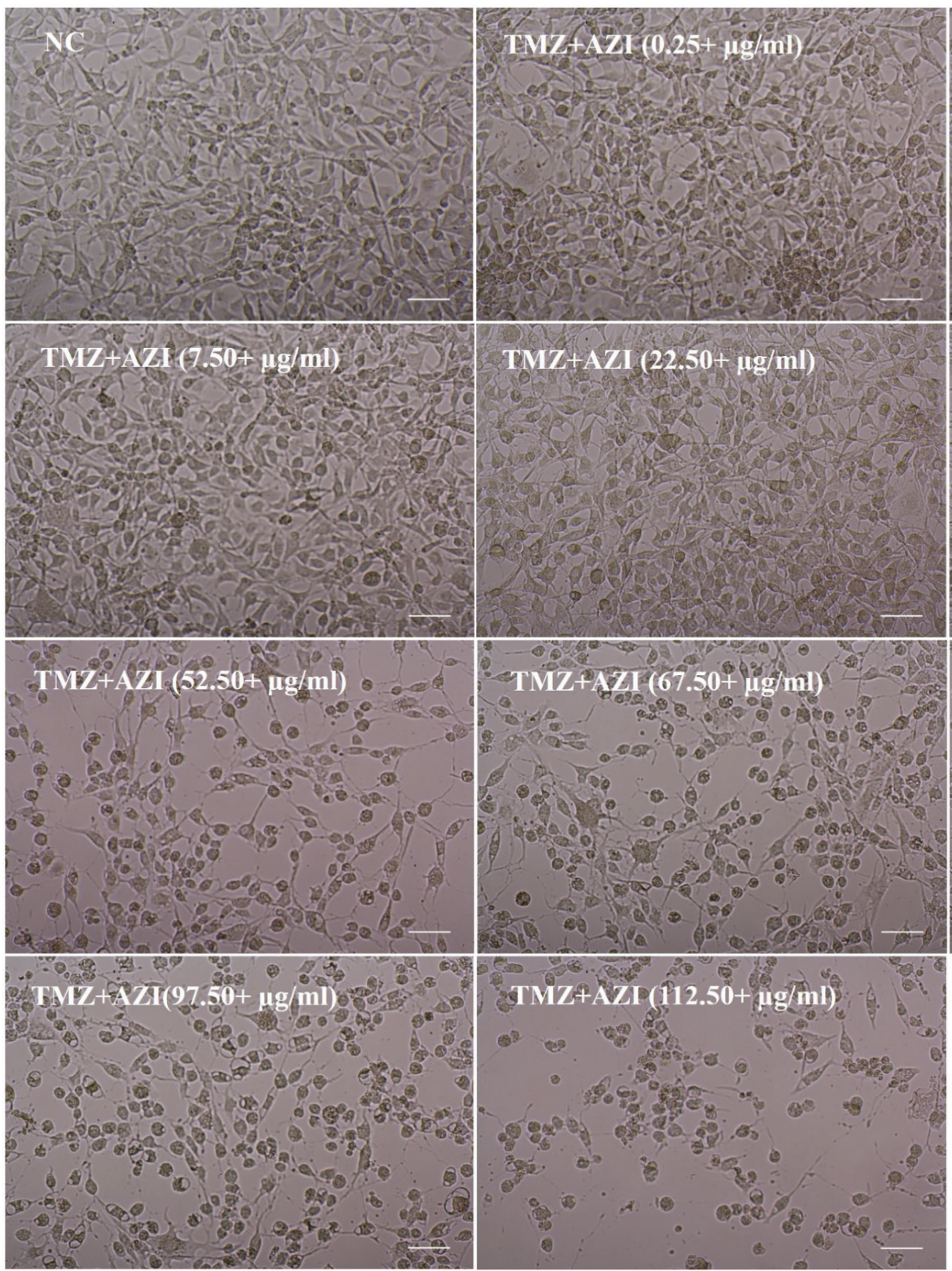

Figure 4. Effect of TMZ + AZI on U87 cells. Microphotographs were taken after 48h of incubation with different concentrations of TMZ + AZI, $(s c a l e ~ b a r s ~=100 ~ \mu m)$.
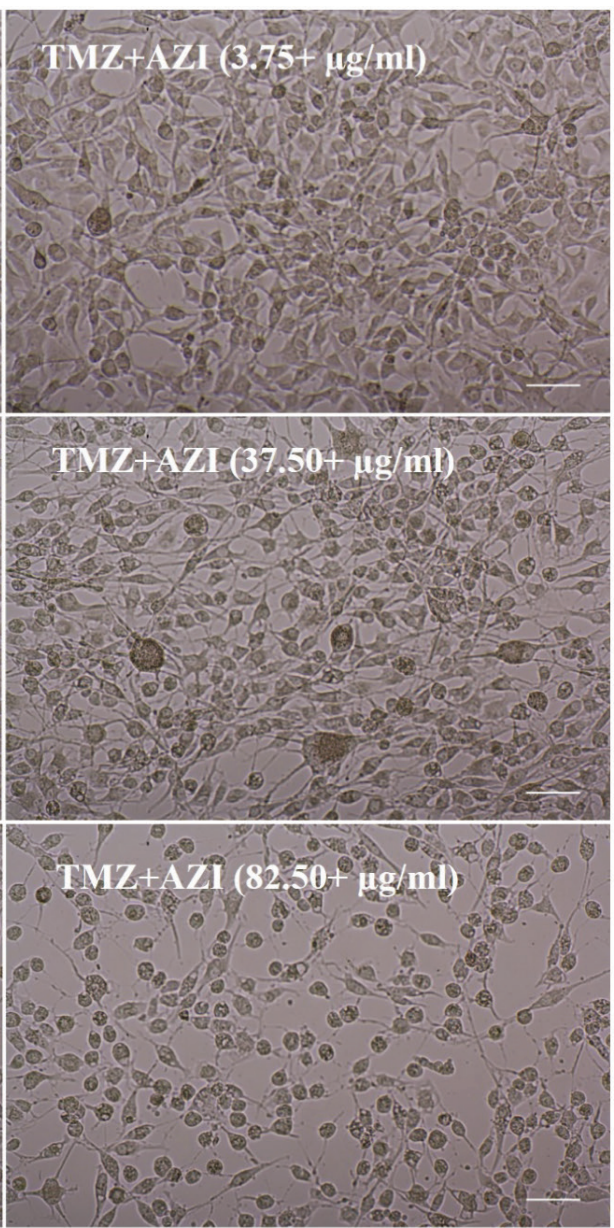